\title{
Direct atomic-scale evidence for shear-dilatation correlation in metallic glasses
}

\author{
Yun-Jiang Wang a,*, M.Q. Jiang a , Z.L. Tian ${ }^{\mathrm{a}}$, L.H. Dai ${ }^{\mathrm{a}, \mathrm{b}, * *}$ \\ a State Key Laboratory of Nonlinear Mechanics, Institute of Mechanics, Chinese Academy of Sciences, Beijing 100190, China \\ b State Key Laboratory of Explosion Science and Technology, Beijing Institute of Technology, Beijing 100081, China
}

\section{A R T I C L E I N F O}

Article history:

Received 10 August 2015

Received in revised form 4 September 2015

Accepted 4 September 2015

Available online 18 September 2015

\section{Keywords:}

Metallic glasses

Shear-dilatation

Molecular dynamics

Density functional theory

\begin{abstract}
A B S T R A C T
Direct atomic-scale evidence is presented for the shear-dilatation correlation in metallic glasses via molecular dynamics and first-principles calculations. A quantitative parabolic relationship is established between the atomic local shear and hydrostatic volumetric strains by carrying out statistical analysis on a deformed glass model. The correction is further verified by density functional theory. Our atomistic demonstration of shear-dilatation correlation collaborates with the experimentally observed a few percent volume change in shear bands. It brings quantitative insights into the unique correlation between shear transformation and cavitation in metallic glasses.
\end{abstract} (c) 2015 Elsevier Ltd. All rights reserved.
Metallic glasses (MGs) are a category of promising high strength structural materials with many other superior physical and chemical properties [1]. The deformation mechanisms of such amorphous solid state are in sharp contrast with their crystalline counterparts since there are no long-range atoms packing pattern in MGs [2,3]. No conventional mechanisms, such as ordinary dislocation plasticity or deformation twinning in crystals, exist in MGs which can carry plastic deformation. Therefore MGs usually suffer from the notorious catastrophic failure with a strong strain localization (shear banding) phenomenon $[4,5]$.

Several models have been proposed to rationalize the inelastic deformation of MGs, which include free volume [6], shear transformation zone (STZ) [7-9], cooperative shear model (CSM) [10,11], flow unit [12], vibrational soft spot [13], shear-diffusive transformation [14,15], and our recently proposed tensile transformation zone (TTZ) [16-18]. All of these models are based on the local structural rearrangement of atoms via the interplay of atomic-scale shear and dilation/contraction in MGs [19-24]. Among them, Argon's STZ model involves local rearrangement of a cluster of atoms undergoing a stress-driven, and thermally activated shear distortion $[25,23,24]$. Whereas Spaepen's free volume model is based on a dynamic equilibrium between the stress-assisted creation and annihilation of free volume [6]. The free volume behaviors can be regarded as dilation and contraction of local atomic environments. Since both models have been successfully adopted to describe the homogeneous and inhomogeneous flows in MGs, there should be some

\footnotetext{
* Corresponding author

** Correspondence to: L. H. Dai, State Key Laboratory of Nonlinear Mechanics, Institute of Mechanics, Chinese Academy of Sciences, Beijing 100190, China.

E-mail addresses: yjwang@imech.ac.cn (Y.-J. Wang), lhdai@Inm.imech.ac.cn (L.H. Dai).
}

intrinsic correlation between shear and dilatation/compaction during deformation of glasses [21,26].

Generally, shear-dilatation correlation is an intrinsic nature of deformation in amorphous alloys although local compaction is also allowed $[27,28]$. For example, intuitively derived law between shear strain and local dilatation has been used in constitutive modelings of MGs [29]. Shear is not necessarily the only deformation mode accommodating the local atomic rearrangement. The microscopic scenario is that the STZ operations redistribute stress spatially which usually leads to the creation of free volume via atomic-scale dilatation $[21,1]$. So that local structural dilatation $[19,30,31]$, density change [27,28], and even nanovoids $[32,33,21,34]$ have been observed within the shear bands of MGs. Although the concept of shear-dilatation correlation is widely accepted in the glass community, and various experiments and simulations have indicated STZ and cavitation as important deformation and fracture mechanisms of glassy alloys [33,35,31,18,17,34], there still lacks a direct microscopic evidence and no quantitative relationship established for such an intimate correlation. Whereas a direct observation and derivation of such a quantitative correlation [19] down to atomic-scale is usually an extreme challenging task in experiments [36].

To this end, we conduct combined molecular dynamics (MD) and density functional theory (DFT) calculations to confirm the sheardilatation correlation in MGs. By statistical analysis on the atomicscale strains, we establish a parabolic relationship between the atomic shear and volumetric strains in MGs both spatially and temporally. The atomic information provides quantitative insights into the intrinsic deformation and fracture characteristics of MGs.

To establish the shear-dilatation correlation, we conduct statistical analysis on a deformed $\mathrm{Cu}_{50} \mathrm{Zr}_{50}$ model glass described by a Finnis- 
Sinclair type potential [37]. The model contains 100,000 atoms which allows a reliable statistical analysis on the atomic strains. It is prepared by a heating-quenching technique from liquid to glass state with a cooling rate of $10^{10} \mathrm{~K} / \mathrm{s}$. The MD is performed by LAMMPS code [38]. Pure shear deformation is applied to induce the atomic strains. The strain rate is $\dot{\gamma}=10^{6} \mathrm{~s}^{2}$, and temperature is $50 \mathrm{~K}$. Such simulation conditions allow sufficient structural relaxation which eventually yields shear banding. The DFT calculations are carried out by VASP code [39] with a model containing 256 atoms. The generalized gradient approximation (GGA) of projector augmented wave (PAW) is adopted for parametrization of the exchange-correlation functional [40]. Athermal quasi-static shear is utilized to distort the atoms. During shear, all the components of stress tensor are relaxed to a negligible values except the shear direction [41]. The atoms are fully relaxed until the Hellmann-Feynman force is smaller than $0.01 \mathrm{eV} / \mathrm{A}$. The visualization is done by OVITO software [42].

To characterize the local distortion at atomic-scale, we use three definitions of atomic strains. First, the atomic local shear strain (local von Mises strain) is defined as [43]

$\eta^{\text {Mises }}=\sqrt{\eta_{y z}^{2}+\eta_{x z}^{2}+\eta_{x y}^{2}+\frac{\left(\eta_{y y}-\eta_{z z}\right)^{2}+\left(\eta_{x x}-\eta_{z z}\right)^{2}+\left(\eta_{y y}-\eta_{y y}\right)^{2}}{6}}$,

where $\eta_{x x}, \ldots$, are the components of local Lagrangian strain matrix. Then, the atomic hydrostatic volumetric strain is consequently written as [43]

$\delta=\frac{\Delta V}{V} \approx \eta_{x x}+\eta_{y y}+\eta_{z z}$

which is used to denote atomic-scale dilatation. $V$ denotes the volume of the simulation box, and $\Delta V$ is the variation. Finally, the local minimum non-affine squared displacement

$D_{\min }^{2}=\frac{1}{N} \sum_{j}\left\{\vec{r}_{j}(t)-\vec{r}_{i}(t)-\boldsymbol{J}_{i}\left[\vec{r}_{j}(t-\Delta t)-\vec{r}_{i}(t-\Delta t)\right]\right\}^{2}$ defined by Falk and Lager [8] is also a good measure of inelastic deformation. Here the atom $i$ is surround by $j \in N$ atoms. $\vec{r}$ is the position of atoms. $J_{i}$ is the local deformation gradient. $t$ and $\Delta t$ denote time and time interval.

Fig. 1 shows the analysis of spatial strain distribution on a deformed MD model at macroscopic shear strain of $\gamma=0.12$. The stress-strain curve shown in Fig. 1(a) indicates the appearance of shear localization at such strain magnitude. Fig. 1(b) plots the distributions of $\eta^{\text {Mises }}$, $D_{\min }^{2}$, and $\delta$ for all 100,000 atoms, respectively. Most of the atoms have experienced atomic shear strain less than 0.1 with the peak at $\eta^{\text {Mises }} \sim 0.06$. Whereas there is a very wide distribution of $D_{\mathrm{min}}^{2}$. Most of them are less than $1 \AA^{2}$. Because we apply pure shear deformation, the atomic volumetric strain distributes almost symmetrically around $\delta=0$. If all the atoms are colored according to their atomic strains, as shown in Fig. 1(c), we can observe good spatial correspondence among them. It indicates that there is indeed strong correlation between shear, non-affine displacement, and dilatation at atomic-scale. It is also interesting to notice both atomic dilatation and contraction if one carefully check the volumetric coloring scheme, which agrees with latest experimental observations [27,28]. In Fig. 1(d) we bin and reduce the atomic strain along the $y$ direction. It is noticed that the mostly strained atoms locate at around $y \sim 10 \AA$, which is actually the location of shear banding. Again, the good correspondence illustrates the spatial shear-dilatation correlation in this model glass. Note that in Fig. 1(b), we plot the distribution of local dilation for all the atoms which seems to be symmetric about 0.0 but with slight deviation to the positive strain. It indicates that compaction also occurs at some regions. However, if we coarse-grain the volumetric strain spatially with 10 atoms in one unit, as shown in Fig. 1(d), the global effect is dilatation.

To further establish a quantitative relationship between shear and dilatation, we provide a statistical analysis on the atomic strains with all the 100,000 data points. The results are summarized in Fig. 2, in which Fig. 2(a) demonstrates the key results. For a first approximation, all the raw MD data (gray crosses) indicate some positive correlation between

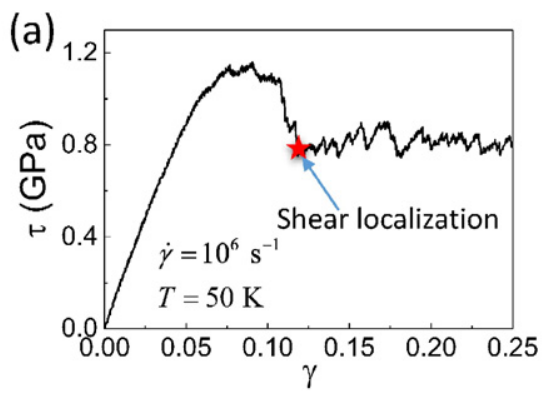

(b)

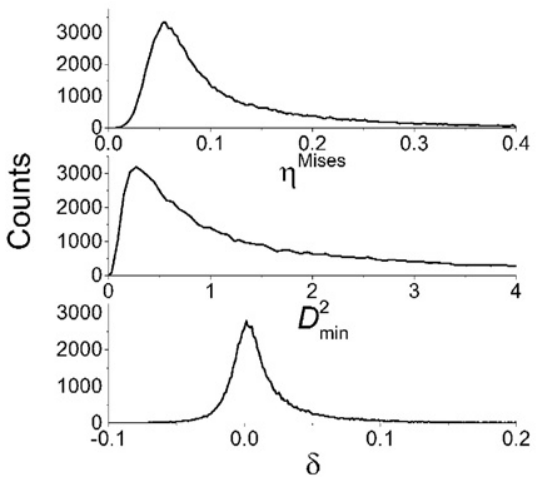

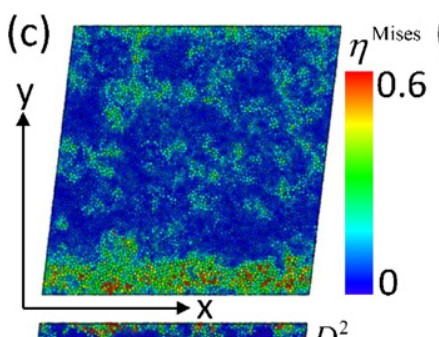
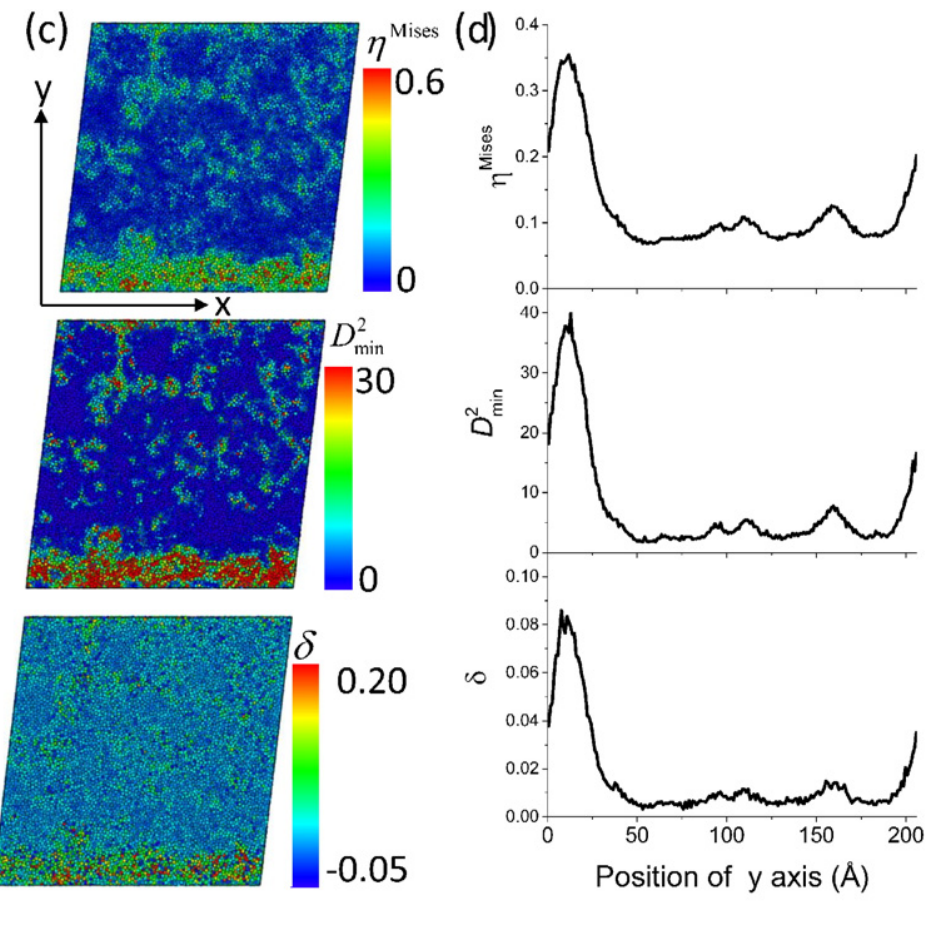

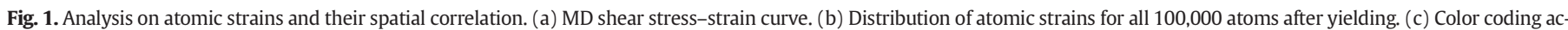
cording to $\eta^{\text {Mises }}, D_{\min }^{2}$, and $\delta$, respectively. (d) Spatial correspondence among different atomic strains $\eta^{\text {Mises }}, D_{\min }^{2}$, and $\delta$. 

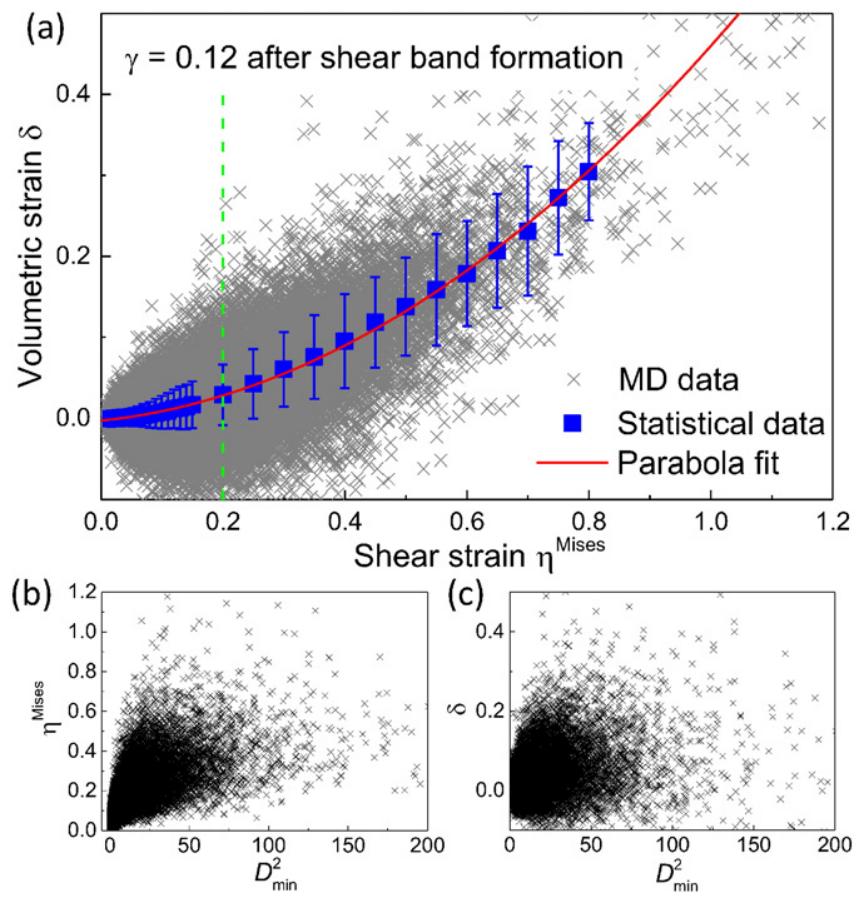

Fig. 2. Quantitative shear-dilatation correlation. (a) Correlation between atomic local shear strain and volumetric strain. A statistical analysis reveals a parabolic relationship, i.e., $\delta=a_{1} \eta^{\text {Mises }}+a_{2}\left(\eta^{\text {Mises }}\right)^{2}$, with $a_{1}=0.078 \pm 0.007$, and $a_{2}=0.384 \pm 0.011$. The gray crosses are the raw MD data. The blue squares are the average atomic strains, and error bars indicate the standard deviation. The vertical broken line denotes the $\eta^{\text {Mises }}$ criterion for severely deformed atoms. (b) Correlation between $\eta^{\text {Mises }}$ and $D_{\min }^{2}$. (c) Correlation between $\delta$ and $D_{\text {min }}^{2}$.

local atomic shear strain and volumetric strain. We then make a statistical analysis on these data with a bin window of 0.01 in $\eta^{\text {Mises }}$ and $\delta$. The statistical average shear-volumetric data are shown as blue squares in Fig. 2(a). The error bars denote standard deviations. It is quite interesting to notice a simple parabolic relationship between $\eta^{\text {Mises }}$ and $\delta$, i.e.,

$\delta=a_{1} \eta^{\text {Mises }}+a_{2}\left(\eta^{\text {Mises }}\right)^{2}$,

with $a_{1}=0.078 \pm 0.007$, and $a_{2}=0.384 \pm 0.011$. Such a parabolic relationship concisely quantifies the shear-dilatation correlation in MGs down to atomic-scale. The positive correlation is qualitatively consistent with Argon's assumption of linear relationship between shear and dilatation in shear bands [19]. Note that the exact number of the coefficients in Eq. (4), or the level of correlation, could be materials dependent since the shear-to-bulk modulus ratio $(G / B)$, or Poisson ratio, varies with compositions [44]. The quantitative information is meaningful to understand the brittle-ductile behavior of a glass since it is closely related to the Poisson criterion [44]. For the present 'ductile' CuZr glass with smaller $G / B$ ratio, the correlation is relatively weaker which indicates that shear could be the dominating deformation mode [35]. While it is most likely to find brittle behaviors in stronger shear-dilatation correlation glass (with bigger $G / B$ ratio) since the great propensity to nucleate cavitation (or TTZ) via shear-induced dilatation, which has been proposed as a brittle fracture mechanism in MGs $[33,35,31,18,17,34]$. For comparison, we also plot the $\eta^{\text {Mises }}-D_{\min }^{2}$, and $\delta-D_{\text {min }}^{2}$ data in Figs. 2(b) and (c), respectively. It is clear to see strong positive correlation between $\eta^{\text {Mises }}$ and $D_{\mathrm{min}}^{2}$, although the latter includes both shear and volumetric components of inelastic deformation. Consequently, there also exists positive correlation between $\delta$ and $D_{\min }^{2}$, as demonstrated by Fig. 2(c).

The spatial shear-dilatation correlation also holds from a temporal point of view. Fig. 3(a) shows the atomic strains as a function of
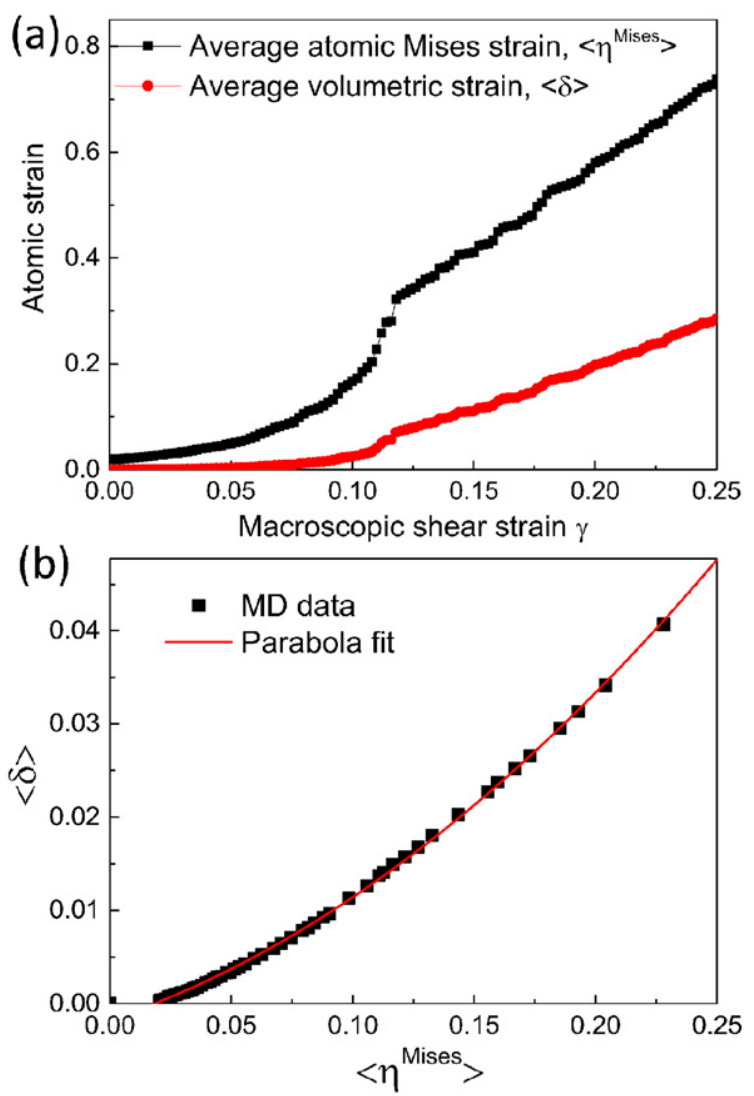

Fig. 3. Shear-dilations correlation in the severely deformed regions during deformation process. (a) Average atomic local shear strain and volumetric strain as a function of macroscopic strain during shear deformation. (b) Parabolic relationship between average atomic shear and volumetric strain during deformation, $\delta=$ $(0.088 \pm 0.003) \eta^{\text {Mises }}+(0.439 \pm 0.014)\left(\eta^{\text {Mises }}\right)^{2}$.

macroscopic strain during the shear deformation. Here the statistics are only performed on those atoms experiencing atomic shear strain larger than 0.2 (Fig. 2(a)), which are actually those atoms in the STZs or the shear band, as shown in the highlighted regions of Fig. 1(c). We notice the evolution of $\eta^{\text {Mises }}$ and $\delta$ follows the same trend. Abrupt increases in atomic strains appear at $\gamma=0.11-0.12$ where shear band forms. A close look indicates the mean atomic volumetric strain reaches $4 \%-7 \%$ during the initial shear band formation. The calculated dilatation is consistent with experiments where a few percent volume change is associated with shear band initiation $[30,45,28]$. Note that the shear-induced dilatation could be probably enhanced in brittle glasses, where the dilation can be as much as $10 \%$ which may eventually lead to fracture via cavitation (or TTZ) mechanisms [35,31]. For a detailed examination, the average atomic volumetric strain is plotted against average atomic local shear strain in Fig. 3(b). Similar to Eq. (4), a parabolic relationship, $\delta=$ $(0.088 \pm 0.003) \eta^{\text {Mises }}+(0.439 \pm 0.014)\left(\eta^{\text {Mises }}\right)^{2}$, can describe the shear-dilatation during deformation fairly well. So that we confirm the shear-dilatation correlation during deformation as well as the static spatial correlation summarized in Figs. 1-2.

Finally, we conduct DFT shear calculations on the same $\mathrm{Cu}_{50} \mathrm{Zr}_{50}$ system to support the MD findings. The results are displayed in Fig. 4. The DFT shear stress-strain curve is shown in Fig. 4(a). Notice that the model is too small to allow any shear banding during deformation. Instead, the yielding is governed by the uniform stretching of metallic bonds. Therefore the critical stress and strain could be regarded as 'ideal strength' and 'ideal strain' of the present CuZr glass. Such an athermal shear strength is as high as $\tau_{\text {ideal }}=2.8 \mathrm{GPa}$, and the material can be sheared as far as $\gamma_{\text {ideal }}=0.15$ if there is no emergence of shear band. Note that the 'ideal strength' here should be smaller than the true value since computational models stay at higher position of the potential 

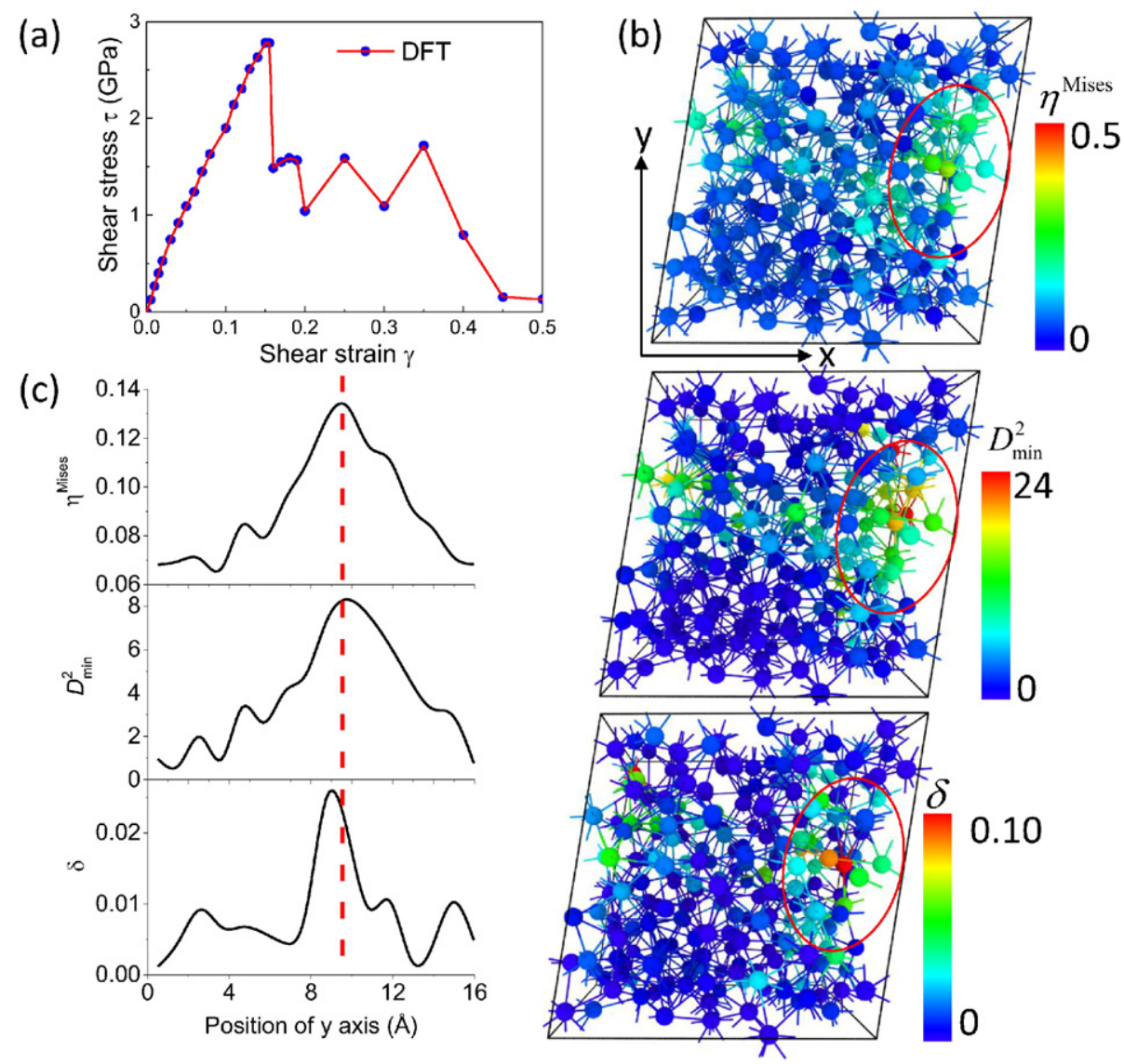

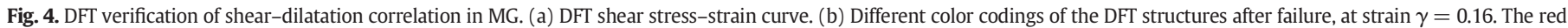
ellipses denote the severely deformed regions. (c) Spatial correlation among different atomic strains.

energy landscape which presents small energy barriers. For example, the DFT estimated shear modulus $G=27 \mathrm{GPa}$ is smaller than the experimental value $31 \mathrm{GPa}$ [46]. To examine the shear-dilatation correlation, we choose the model just after failure (at $\gamma=0.16$ ) to analyze the atomic strains because large atomic distortion happens at this moment.

Similar to Fig. 1(c), we also visualize the DFT structures at $\gamma=0.16$ by color coding their atomic strains defined by Eqs. (1)-(3). It is noticed that all the severely distorted atoms are located in the same region, which is denoted by the ellipses. This local bond breaking region may be thought as a STZ. There are nice correspondences between these atomic strains in describing the STZ. The correspondence actually indicates a shear-dilatation correlation in the STZ region. For a detailed demonstration, we also bin and reduce the atomic strains along the $y$ direction as shown in Fig. 4(c). It is shown that all the peaks appear at similar position of $10 \AA$ in the $y$ axis. Moreover, the peak strain data $\left(\eta^{\text {Mises }}=0.135, \delta=0.023\right)$ almost lie at the theoretical prediction of Eq. (4) $\left(\delta=0.018\right.$ at $\left.\eta^{\text {Mises }}=0.135\right)$. This is in turn a solid support for the quantitative shear-dilatation correlation established by MD.

To summarize, we confirm and establish a quantitative relationship between atomic-scale shear and dilatation in amorphous alloys by carrying out statistics on a deformed MD sample. A simple parabolic relationship is revealed from both spatial and temporal point of views. The MD findings are further clarified by DFT athermal quasi-static shear calculations. The atomistically deduced atomic dilatation accompanying shear band initiation reaches a few percent, which agrees with experimental observations $[30,45,28]$. The atomic-scale information for the shear-dilatation correlation presented here bridges the STZ and free volume model for deformation of MGs. It is also meaningful to understand the unique shear banding and fracture via cavitation mechanisms of amorphous solids.
The authors acknowledge the financial support from the NSFC (nos. $11402269,11372315,11472287,11132011)$, the National Key Basic Research Program of China (no. 2012CB937500), and the CAS/SAFEA International Partnership Program for Creative Research Teams.

\section{References}

[1] C. Schuh, T. Hufnagel, U. Ramamurty, Acta Mater. 55 (2007) 4067-4109, http:// dx.doi.org/10.1016/j.actamat.2007.01.052 (URL: http://linkinghub.elsevier. com/retrieve/pii/S135964540700122X).

[2] Y. Cheng, E. Ma, Prog. Mater. Sci. 56 (2010) 379-473, http://dx.doi.org/10.1016/ j.pmatsci.2010.12.002 (URL: http://linkinghub.elsevier.com/retrieve/pii/ S0079642510000691)

[3] A. Hirata, P. Guan, T. Fujita, Y. Hirotsu, A. Inoue, A.R. Yavari, T. Sakurai, M. Chen, Nat. Mater. 9 (2010) 1-6, http://dx.doi.org/10.1038/nmat2897 (URL: http://dx.doi.org/ 10.1038/nmat2897).

[4] B. Dodd, Y. Bai, Adiabatic Shear Localization: Frontiers and Advances, Elsevier, 2012 (URL: http://store.elsevier.com/Adiabatic-Shear-Localization/isbn-9780080982007/).

[5] A. Greer, Y. Cheng, E. Ma, Mater. Sci. Eng. R. Rep. 74 (2013) 71-132, http://dx.doi org/10.1016/j.mser.2013.04.001 (URL: http://linkinghub.elsevier.com/retrieve/pii/ S0927796X13000259).

[6] F. Spaepen, Acta Metall. 25 (1977) 407-415, http://dx.doi.org/10.1016/00016160(77)90232-2 (URL: http://www.sciencedirect.com/science/article/pii/ 0001616077902322).

[7] A.S. Argon, Acta Metall. 27 (1979) 47-58, http://dx.doi.org/10.1016/00016160(79)90055-5 (URL: http://www.sciencedirect.com/science/article/pii/ 0001616079900555).

[8] M. Falk, J. Langer, Phys. Rev. E 57 (1998) 7192-7205, http://dx.doi.org/10.1103/ PhysRevE.57.7192 (URL: http://link.aps.org/doi/10.1103/PhysRevE.57.7192).

[9] D. Pan, A. Inoue, T. Sakurai, M.W. Chen, Proc. Natl. Acad. Sci. U. S. A. 105 (2008) 14769-14772, http://dx.doi.org/10.1073/pnas.0806051105 (URL: http://www. pubmedcentral.nih.gov/articlerender.fcgi?artid $=2567442 \&$ tool $=$ pmcentrez\& rendertype $=$ abstract $)$.

[10] W.L. Johnson, K. Samwer, Phys. Rev. Lett. 95 (2005) 195501, http://dx.doi.org/10.1103/ PhysRevLett.95.195501 (URL: http://link.aps.org/doi/10.1103/PhysRevLett.95.195501).

[11] L. Sun, M.Q. Jiang, L.H. Dai, Scr. Mater. 63 (2010) 945-948, http://dx.doi.org/10. 1016/j.scriptamat.2010.07.011. 
[12] Z. Lu, W. Jiao, W.H. Wang, H.Y. Bai, Phys. Rev. Lett. 113 (2014) 045501, http://dx. doi.org/10.1103/PhysRevLett.113.045501 (URL: http://link.aps.org/doi/10. 1103/PhysRevLett.113.045501).

[13] J. Ding, S. Patinet, M.L. Falk, Y. Cheng, E. Ma, Proc. Natl. Acad. Sci. U. S. A. 111 (2014) 14052-14056, http://dx.doi.org/10.1073/pnas.1412095111 (URL: http://www.pnas. org/cgi/doi/10.1073/pnas.1412095111).

[14] C.-C. Wang, Y.-W. Mao, Z.-W. Shan, M. Dao, J. Li, J. Sun, E. Ma, S. Suresh, Proc. Natl. Acad. Sci. U. S. A. 110 (2013) 19725-19730, http://dx.doi.org/10.1073/pnas. 1320235110 (URL: http://www.pubmedcentral.nih.gov/articlerender.fcgi?artid= 3856821\&tool $=$ pmcentrez\&rendertype $=$ abstract).

[15] Y. Mao, J. Li, Y.-C. Lo, X. Qian, E. Ma, Phys. Rev. B 91 (2015) 214103, http://dx.doi.org/ 10.1103/PhysRevB.91.214103 (URL: http://link.aps.org/doi/10.1103/PhysRevB.91. 214103).

[16] M.Q. Jiang, Z. Ling, J.X. Meng, L.H. Dai, Philos. Mag. 88 (2008) 407-426, http://dx.doi. org/10.1080/14786430701864753.

[17] X. Huang, Z. Ling, L.H. Dai, J. Appl. Phys. 116 (2014) 143503, http://dx.doi.org/10. 1063/1.4897552 (URL: http://scitation.aip.org/content/aip/journal/jap/116/14/10 1063/1.4897552).

[18] M.Q. Jiang, G. Wilde, J.H. Chen, C.B. Qu, S.Y. Fu, F. Jiang, L.H. Dai, Acta Mater. 77 (2014) 248-257, http://dx.doi.org/10.1016/j.actamat.2014.05.052 (URL: http:// linkinghub.elsevier.com/retrieve/pii/S135964541400408X).

[19] A.S. Argon, J. Megusar, N.J. Grant, Scr. Metall. 19 (1985) 591-596, http://dx.doi.org/10. 1016/0036-9748(85)90343-6 (URL: http://www.sciencedirect.com/science/article/ pii/0036974885903436).

[20] A. Lemaître, Phys. Rev. Lett. 89 (2002) 195503, http://dx.doi.org/10.1103/PhysRevLett. 89.195503 (URL: http://link.aps.org/doi/10.1103/PhysRevLett.89.195503).

[21] R. Bhowmick, R. Raghavan, K. Chattopadhyay, U. Ramamurty, Acta Mater. 54 (2006) 4221-4228, http://dx.doi.org/10.1016/j.actamat.2006.05.011.

[22] A. Lematre, Phys. Rev. Lett. 113 (2014) 245702, http://dx.doi.org/10.1103/PhysRevLett 113.245702 (URL: http://link.aps.org/doi/10.1103/PhysRevLett.113.245702).

[23] Y. Fan, T. Iwashita, T. Egami, Nat. Commun. 5 (2014) 5083, http://dx.doi.org/10.1038/ ncomms6083 (URL: http://www.nature.com/doifinder/10.1038/ncomms6083).

[24] Y. Fan, T. Iwashita, T. Egami, Phys. Rev. Lett. 115 (2015) 045501, http://dx.doi.org/10 1103/PhysRevLett.115.045501 (URL: http://link.aps.org/doi/10.1103/PhysRevLett. 115.045501).

[25] C.A. Schuh, A.C. Lund, Nat. Mater. 2 (2003) 449-452, http://dx.doi.org/10.1038/ nmat918 (URL: http://www.ncbi.nlm.nih.gov/pubmed/12792648).

[26] M. Jiang, G. Wilde, L. Dai, Mech. Mater. 81 (2015) 72-83, http://dx.doi.org/10 1016/j.mechmat.2014.10.002 (URL: http://linkinghub.elsevier.com/retrieve/ pii/S0167663614001823).

[27] H. Rösner, M. Peterlechner, C. Kübel, V. Schmidt, G. Wilde, Ultramicroscopy 142 (2014) 1-9, http://dx.doi.org/10.1016/j.ultramic.2014.03.006 (URL: http://dx.doi. org/10.1016/j.ultramic.2014.03.006).

[28] V. Schmidt, H. Rösner, M. Peterlechner, G. Wilde, P.M. Voyles, Phys. Rev. Lett. 115 (2015) 035501, http://dx.doi.org/10.1103/PhysRevLett.115.035501 (URL: http:// link.aps.org/doi/10.1103/PhysRevLett.115.035501).
[29] P. Thamburaja, J. Mech. Phys. Solids 59 (2011) 1552-1575, http://dx.doi.org/10. 1016/j.jmps.2011.04.018 (URL: http://dx.doi.org/10.1016/j.jmps.2011.04.018),

[30] D. Klaumünzer, A. Lazarev, R. Maaß, F.H. Dalla Torre, A. Vinogradov, J.F. Löffler, Phys. Rev. Lett. 107 (2011) 1-5, http://dx.doi.org/10.1103/PhysRevLett.107.185502.

[31] P. Guan, S. Lu, M.J.B. Spector, P.K. Valavala, M.L. Falk, Phys. Rev. Lett. 110 (2013) 185502, http://dx.doi.org/10.1103/PhysRevLett.110.185502 (URL: http://link.aps. org/doi/10.1103/PhysRevLett.110.185502).

[32] J. Li, F. Spaepen, T.C. Hufnagel, Philos. Mag. A 82 (2002) 2623-2630, http:/dx.doi. org/10.1080/01418610210152792 (URL: http://www.tandfonline.com/doi/abs/10. 1080/01418610208240056).

[33] W.H. Jiang, F.E. Pinkerton, M. Atzmon, Acta Mater. 53 (2005) 3469-3477, http://dx. doi.org/10.1016/j.actamat.2005.04.003.

[34] Y. Shao, G.-N. Yang, K.-F. Yao, X. Liu, Appl. Phys. Lett. 105 (2014) 181909, http://dx. doi.org/10.1063/1.4901281 (URL: http://scitation.aip.org/content/aip/journal/apl/ 105/18/10.1063/1.4901281).

[35] P. Murali, T. Guo, Y. Zhang, R. Narasimhan, Y. Li, H.J. Gao, Phys. Rev. Lett. 107 (2011) 1-5, http://dx.doi.org/10.1103/PhysRevLett.107.215501 (URL: http://link.aps.org/ doi/10.1103/PhysRevLett.107.215501).

[36] A. Hirata, L.J. Kang, T. Fujita, B. Klumov, K. Matsue, M. Kotani, A.R. Yavari, M.W. Chen, Science 341 (2013) 376-379, http://dx.doi.org/10.1126/science.1232450 (URL: http://www.ncbi.nlm.nih.gov/pubmed/23845945).

[37] M. Mendelev, M. Kramer, R. Ott, D. Sordelet, Philos. Mag. 89 (2009) 109-126, http:// dx.doi.org/10.1080/14786430802570648.

[38] S. Plimpton, J. Comput. Phys. 117 (1995) 1-19, http://dx.doi.org/10.1006/jcph.1995. 1039 (URL: http://dx.doi.org/10.1006/jcph.1995.1039).

[39] G. Kresse, J. Furthmüller, Phys. Rev. B 54 (1996) 11169-11186, http://dx.doi.org/10. 1103/PhysRevB.54.11169 (URL: http://link.aps.org/doi/10.1103/PhysRevB.54.11169).

[40] J.P. Perdew, J.A. Chevary, S.H. Vosko, K.A. Jackson, M.R. Pederson, D.J. Singh, C. Fiolhais, Phys. Rev. B 46 (1992) 6671-6687, http://dx.doi.org/10.1103/PhysRevB. 46.6671 (URL: http://link.aps.org/doi/10.1103/PhysRevB.46.6671).

[41] Y.-J. Wang, C.-Y.Wang, Appl. Phys. Lett. 94 (2009) 261909, http://dx.doi.org/10.1063/1. 3170752 (URL: http://link.aip.org/link/APPLAB/v94/i26/p261909/s1\&Agg=doi).

[42] A. Stukowski, Model. Simul. Mater. Sci. Eng. 18 (2010) 015012, http://dx.doi.org/10. 1088/0965-0393/18/1/015012 (URL: http://stacks.iop.org/0965-0393/18/i=1/a= 015012 ? key = crossref.6895e2c3bb522d1563fb2e2fe9f22789).

[43] F. Shimizu, S. Ogata, J. Li, Mater. Trans. 48 (2007) 2923-2927, http://dx.doi.org/10. 2320/matertrans.MJ200769 (URL: http://www.jim.or.jp/journal/e/48/11/2923.html).

[44] W.H. Wang, Prog. Mater. Sci. 57 (2012) 487-656, http://dx.doi.org/10.1016/j.pmatsci. 2011.07.001 (URL: http:/linkinghub.elsevier.com/retrieve/pii/S0079642511000934).

[45] Y. Shao, K. Yao, M. Li, X. Liu, Appl. Phys. Lett. 103 (2013) 0-4, http://dx.doi.org/10. 1063/1.4826117 (URL: http://scitation.aip.org/content/aip/journal/apl/103/17/10. 1063/1.4826117).

[46] G.J. Fan, M. Freels, H. Choo, P.K. Liaw, JJZ Li, W.K. Rhim, W.L. Johnson, P. Yu, W.H. Wang Appl. Phys. Lett. 89 (2006) 3, http://dx.doi.org/10.1063/1.2408634 (URL: < Go to ISI>:// 000242886500051). 